Acknowledgement: We would like to thank Mr. Kwok MF for his support in acquisition of data.

Disclosure of Interests: None declared

DOI: 10.1136/annrheumdis-2019-eular.966

\section{SAT0439 RENOPROTECTIVE EFFECT OF URATE LOWERING THERAPY IN GOUTY PATIENTS WITH MODERATE CHRONIC KIDNEY DISEASE}

Marta Novella-Navarro ${ }^{1}$, José Luis Cabrera-Alarcón ${ }^{2}$, Francisco AramburuMuñoz ${ }^{3}$, Iustina Janta ${ }^{4}$, Alejandro Prada-Ojeda ${ }^{1}$, Luis Sala-Icardo ${ }^{1}$, Paloma García de la Peña ${ }^{3}$, Enrique Calvo-Aranda ${ }^{5} .{ }^{1}$ Hospital Universitario Torrejon,

Rheumatology, Madrid, Spain; ${ }^{2}$ CNIC, Bioinformatics, GENOPHOS group, Madrid, Spain; ${ }^{3}$ Hospital Universitario HM Sanchinarro, Rheumatology, Madrid, Spain; ${ }^{4}$ Hospital Universitario Gregorio Marañon, Rheumatology, Madrid, Spain; ${ }^{5}$ Hospital Universitario Infanta Leonor, Madrid, Spain

Background: Approximately $25 \%$ of gouty patients suffer from chronic kidney disease (CKD). High serum uric acid (sUA) levels have been related to estimated glomerular filtration-rate (eGFR) imbalance. Beneficial effect of treatment with xanthine oxidase inhibitors (XOI), mostly allopurinol, has already been proved in patients with CKD and asymptomatic hyperuricemia. Although several studies have described the efficacy and renal safety of treatment with $\mathrm{XOI}$ in gout, few authors have analyzed its effect on GFR in gouty patients with moderate CKD.

Objectives: To assess the effect of $\mathrm{XOI}$ therapy in gouty patients with moderate $\mathrm{CKD}$, in terms of eGFR changes.

Methods: In this multicenter, retrospective study, we included patients from 4 centers diagnosed with gout (EULAR/ACR criteria) and stage-3 CKD according to Cockroft-Gault formula (eGFR $30-59 \mathrm{ml} / \mathrm{min} / \mathrm{m}^{2}$ ) who received XOI (febuxostat and allopurinol) with a follow-up for 6 and 12 months. We used clinical records to collect patient features (age, sex, body mass index, sUA, hypertension (HTA), diabetes mellitus (DM), dyslipidemia (DL), cardiovascular events), treatments (lipid-lowering drugs, antihypertensives, antidiabetics, antiplatelet therapy, NSAIDs, urate lowering treatments and colchicine) and gout history (duration of disease, tophi presence, clinical and ultrasonographic (US) pattern (monoarticular, oligoarticular, polyarticular).

Statistical analysis: descriptive analysis of variables. Mixed effects model lineal regression Differences were considered significant $p<0.05$.

Results: 52 patients with gout and stage-3 CKD were identified. We obtained complete 6 and 12-months follow-up from 37 patients (33 males and 4 females). Mean age was $74.11 \pm 6.96$ years, $32.4 \%$ DM, $83.78 \%$ HTA, $56 \%$ DL, $40 \%$ tophaceous gout, Clinical and US pattern $(37.8 \%$ polyarticular, 37.8 oligoarticular and $24.3 \%$ monoarticular). Febuxostat 19 patients, Allopurinol 18. Mean baseline sUA was $8.63 \pm 1.33 \mathrm{mg} / \mathrm{dl}$, and baseline eGFR was $47.77 \pm 8.45 \mathrm{ml} / \mathrm{min} / \mathrm{m}^{2}$. To assess the effects of considered variables over eGFR a linear mixed model was adjusted using nlme R-package. Within the adjusted model we obtained significant differences in eGFR between baseline and 6 months $(p=0.0081)$, and between baseline and 12-months $(p=0.0028)$. sUA decreased significantly between baseline and $6(p=0.0181)$ and 12 months $(p=0.0188)$.

Conclusion: Reduction of sUA levels in gouty patients with $\mathrm{XOI}$ entitles an improvement of eGFR in stage-3 CKD. These findings suggest that the response to urate lowering therapy take place in the first 6 months, leading to an improvement in eGFR in this period. From 6 months to 1 year, sUA levels are stabilized and so is eGFR.

\section{REFERENCES}

[1] Tanaka K, Nakayama M, Kanno M, et al. Renoprotective effects of febuxostat in hyperuricemic patientswith chronic kidney disease: a parallel-group, randomized, controlled trial. Clin Exp Neprhol 2015

[2] Juge PA, Truchetet ME, Pillebout E, et al. Efficacy and safety of febuxostat in 73 gouty patients with stage $4 / 5$ chronic kidney disease. A retrospective study of 10 centers. Joint Bone Spine (2016)

Disclosure of Interests: Marta Novella-Navarro: None declared, José Luis Cabrera-Alarcón: None declared, Francisco Aramburu-Muñoz: None declared, lustina Janta: None declared, Alejandro Prada-Ojeda: None declared, Luis Sala-Icardo: None declared, Paloma García de la Peña: None declared, Enrique Calvo-Aranda Speakers bureau: Grühnental, SOBI, Menarini

DOI: 10.1136/annrheumdis-2019-eular.2362

\section{SAT0440 \\ DOES MONOSODIUM URATE DEPOSITION IN GOUT PATIENTS ALTER THE BIOCHEMICAL PROPERTIES OF MENISCAL FIBROCARTILAGE AND HYALINE CARTILAGE? A DUAL-ENERGY COMPUTED TOMOGRAPHY STUDY}

Tristan Pascart ${ }^{1}$, Claire Marzin ${ }^{1}$, Laurène Norberciak ${ }^{1}$, Julie Legrand ${ }^{1}$,

Fabio Becce ${ }^{2}$, Jean-François Budzik'. ${ }^{1}$ Saint-Philibert Hospital, Lille, France;

${ }^{2}$ CHUV Lausanne, Lausanne, Switzerland

Background: Dual-energy computed tomography (DECT) is increasingly used in gout to assess monosodium urate (MSU) crystal deposition in soft tissues. In contrast to ultrasound (US) with its typical double-contour (DC) sign, DECT seems unable to identify MSU deposition deep within joints, where flares occur. DECT has recently shown its potential for discriminating between the various crystal types owing to their biochemical signature.

Objectives: We aimed to assess whether DECT attenuation properties differed between knees of gout patients with and without deep articula MSU deposition characterized by the DC sign on US; more specifically if MSU deposition altered the electron density $(\rho \mathrm{e})$ of various knee structures.

Methods: Consecutive patients with gout were included in this cross-sectional study and their knee MSU burden was assessed using combined DECT and US. Knees were assigned to either DC+ or DC- groups depending on the presence/absence of the DC sign on US. Regions of interest (ROI) were drawn in the following knee zones on a specific coronal DECT image: hyaline cartilage of the patellofemoral and media and lateral tibiofemoral joint spaces, as well as medial and lateral menisci. Regions of interest that exhibited chondrocalcinosis were excluded. Five DECT parameters were obtained: CT numbers (HU) at 80 and $140 \mathrm{kV}$, dual-energy index (DEI), electron density $(\rho \mathrm{e})$, and effective atomic number (Zeff). Knee zones were compared between groups using mixed linear models.

Results: A total of 115 patients were included. Gout duration was 9.8 \pm 9.0 years, mean serum urate was $7.3 \pm 2.3 \mathrm{mg} / \mathrm{dL}$ and $48(41.7 \%)$ patients were under urate lowering therapy. Out of a total 230 knees, 46 $(20 \%)$ were assigned to the DC+ group. Menisci from DC+ and DCpatients had a mean ( \pm standard deviation) Zeff of $7.5 \pm 0.2$ and $7.6 \pm 0.2$ $(\mathrm{p}=0.49)$, mean $\rho$ e of $77 \pm 14$ and $73 \pm 13 \quad(p=0.15)$ and mean DEI of $0.0003 \pm 0.0036$ and $0.0001 \pm 0.0042(p=1)$, respectively. Hyaline cartilage from $\mathrm{DC}+$ and $\mathrm{DC}$ - patients had a mean Zeff of $7.6 \pm 0.2$ and $7.7 \pm 0.2$ $(p=0.49)$, mean $\rho$ e of $65 \pm 21$ and $60 \pm 18 \quad(p=0.17)$ and mean DEI of $0.0020 \pm 0.0049$ and $0.0025 \pm 0.0043 \quad(p=1)$, respectively. No differences were noted between groups in the patellofemoral joint space.

Conclusion: There is an expected increased electron density $(\rho \mathrm{e})$ in meniscal fibrocartilage and hyaline cartilage of gout patients with MSU deposition, even though DECT measures do not reach statistical significance. Particular attention will be given to patients with high MSU burden (large DC signs on US).

Disclosure of Interests: None declared

DOI: 10.1136/annrheumdis-2019-eular.7313

\section{SAT0441 SKIN ADVERSE EVENTS WITH FEBUXOSTAT IN GOUT PATIENTSWITH PREVIOUS SKIN REACTIONS TO ALLOPURINOL. A MULTICENTRE DESCRIPTIVE STUDY}

Neus Quilis Marti ${ }^{1}$, Paloma Vela-Casasempere ${ }^{2}$, Boris Anthony Blanco Cáceres ${ }^{3}$, Cesar Diaz Torne ${ }^{4}$, Enrique Calvo-Aranda ${ }^{5}$. Francisca Sivera ${ }^{6}$, Alejandro PradaOjeda ${ }^{7}$, Fernando Perez-Ruiz ${ }^{3}$, Eliseo Pascual ${ }^{8}$, Mariano Andres ${ }^{2} .{ }^{1}$ Hospital Universitario Vinalopó, Rheumatology Unit, Elche, Alicante, Spain; ${ }^{2}$ Hospital General Universitario de Alicante, Rheumatology, Alicante, Spain; ${ }^{3}$ Hospital Universitario Cruces, Baracaldo, Rheumatology, Bilbao, Spain; ${ }^{4}$ Hospital de la Santa Creu i Sant Pau, Rheumatology, Barcelona, Spain; ${ }^{5}$ Hospital Sanchinarro, Rheumatology, Madrid, Spain; ${ }^{6}$ Hospital General Universitario de Elda, Rheumatology, Elda, Spain; ${ }^{7}$ Hospital Torrejón, Rheumatology, Madrid, Spain; ${ }^{8}$ Universidad Miguel Hernández, Departamento de Medicina Clínica, Alicante, Spain

Background: Allopurinol is first-line urate-lowering drug (ULD) for patients with gout. However, around $10 \%$ of them refer adverse events, often at skin, which can be severe. Febuxostat is a non-purine selective xanthine oxidase inhibitor, often a therapeutic alternative in this setting, though data regarding safety in those patients with previous cutaneous adverse reactions (CAR) to allopurinol is still limited. Some cases and small 
retrospective series have reported skin adverse events under both allopurinol and febuxostat.

Objectives: To assess the cutaneous safety of febuxostat when used in patients with previous skin reactions to allopurinol.

Methods: A multicentre retrospective, descriptive study performed in seven Rheumatology units around Spain. Crystal-proven gout patients with previous CAR to allopurinol and treatment with febuxostat were selected. Demographic (age, gender), clinical (skin events, liver disease, concomitant thiazides) and laboratory variables (serum urate (SU), glomerular filtration rate) were collected. The primary study variable was the rate of patients developing CAR also with febuxostat. A descriptive analysis with estimation of $95 \%$ confidence interval $(95 \% \mathrm{Cl})$ is presented.

Results: Sixty-seven gout patients with prior allopurinol-related CAR treated with febuxostat were enrolled. Their average age was 68.1 years $(S D \pm 14.8)$, being 49 males $(73.1 \%)$. Thirteen of them were under thiazide treatment $(19.4 \%)$ and average glomerular filtration rate was $66.1 \mathrm{ml} / \mathrm{min}$ $( \pm 24.3)$ when allopurinol was started, at a median dose of allopurinol of $100 \mathrm{mg} /$ day (IQR 50-300) and mean SU of $8.9 \mathrm{mg} / \mathrm{dl}( \pm 1.7)$. The reported CAR under allopurinol were nonspecific in 55 (82.1\%), maculopapular rash in 9 (13.4\%), and Stevens-Johnson's syndrome in $3(4.5 \%)$. Out of 67 patients, 10 developed CAR with febuxostat $(14.9 \%$; $95 \% \mathrm{Cl}$ 8.3-25.3\%): nonspecific in 8 cases, one case of maculopapular rash and other of Stevens-Johnson's syndrome. Median (IQR) glomerular filtration rate, starting dose of febuxostat and SU level were 69.5 (42-87.8), 80 $\mathrm{mg} /$ day $(40-80)$ and $8.2 \mathrm{mg} / \mathrm{dl}(7.15-9.14)$, respectively.

Benzbromarone was initiated in all 10 patients with CAR to both allopurinol and febuxostat, and only one developed a nonspecific rash.

Conclusion: In this multicentre study, around $15 \%$ of patients with prior allopurinol-related CARs also developed them with febuxostat. Further prospective and intervention studies are needed to confirm these results, though caution is recommended when using febuxostat in this subgroup of patients.

Disclosure of Interests: Neus Quilis Marti: None declared, Paloma VelaCasasempere Grant/research support from: UCB, Abbvie, Pfizer, Roche, Bristol-Myer-Squibb (another research, not BIOBADASER related), Consultant for: UCB, Lilly, Pfizer, Roche, Bristol-Myer-Squibb, Speakers bureau: Roche, UCB, MSD, Pfizer, GSK, BMS, Lilly, Boris Anthony Blanco Cáceres: None declared, Cesar Diaz Torne Consultant for: Grünenthal, Speakers bureau: Grünenthal, Enrique Calvo-Aranda Speakers bureau: Grühnental, SOBI, Menarini, Francisca Sivera: None declared, Alejandro Prada-Ojeda: None declared, Fernando Perez-Ruiz Grant/research support from: Asociación reumatólogos de Cruces, Consultant for: Grünenthal Horizon Menarini, Speakers bureau: Grünenthal, Menarini, Fundación Española Reumatologiá, Eliseo Pascual Consultant for: Eliseo Pascual has participated in the boards of Astra Zeneca, Menarini, Grunenthal and Horizon, Speakers bureau: speaking fees from Astra Zeneca and Grunenthal, Mariano Andres: None declared

DOI: 10.1136/annrheumdis-2019-eular.4394

\section{SAT0442 CALCIUM PYROPHOSPHATE CRYSTAL ARTHRITIS DURING HOSPITALIZATIONS: A PROSPECTIVE, CRYSTAL-PROVEN CASE SERIES}

Laura Ranieri ${ }^{1}$, Francisca Sivera ${ }^{2}$, Mariano Andrés ${ }^{1} .{ }^{1}$ Alicante, Hospital General Universitario Alicante, Alicante, Spain, ${ }^{2}$ Hospital General Universitario de Elda, ALICANTE, Spain

Background: Despite having passed more than fifty years after its initial description, essential questions for calcium pyrophosphate (CPP) crystal disease, such as clinical spectrum, diagnosis or management schemes, remain unsolved. Acute flares often occurred during hospitalizations. Scant reports have addressed this common setting for CPP crystal disease, and whether these patients behave similarly to ambulatory cases is unknown.

Objectives: The aim of this work was to describe in a prospective way a crystal-proven case series of patients developing acute CPP crystal arthritis during hospitalizations for another conditions.

Methods: An observational, cross sectional descriptive study was conducted in two Spanish centers from November 2013 to December 2018. A prospective convenience sampling was employed to select patients with crystal-proven CPP acute arthritis seen during hospital admissions. Demographic, clinical and CPP-related variables were collected, and X-rays (pelvis, knees, hands, affected joint when different) and laboratory tests (to rule out associated metabolic conditions) were systematically requested. A descriptive analysis is presented.

Results: 90 episodes of acute CPP arthritis in 87 patients were seen in the study period, with an average age of 81.8 years (SD 7.7), 50.6\% of them men. Approximately $26 \%$ of patients reported prior episodes of arthritis, most of them $(68.4 \%)$ as outpatients. Only three patients were on preventive treatment for CPP arthritis (two on colchicine and one on low dose glucocorticoids). Regarding the acute CPP arthritis during admissions, they were predominantly monoarticular $(81.0 \%)$ and the main involved joints were knee $(46.0 \%)$, wrist $(13.8 \%)$ and ankles $(6.9 \%)$. The reasons for admission were diverse, with a mean of 7.7 days (SD 9.1) from admission to flare. About X-rays, $23.8 \%$ showed no chondrocalcinosis (CC) in the evaluated joints [61/80]. In $57.1 \%$ of patients there was chondrocalcinosis in the affected joint [44/77] and regarding usual joints: $74.3 \%$ in knees [55/74], $51.5 \%$ in triangular carpal ligament [34/66], $25.4 \%$ in metacarpo-phalangeal joints [17/67], $20 \%$ in pubic symphysis [14/70] and $17.6 \%$ in coxofemoral joints [12/68]. A secondary form of osteoarthritis was only seen in 10 patients (12.5\%). About associated metabolic diseases, one case of primary hyperparathyroidism-related hypercalcemia and five cases of hypomagnesemia at the time of the flare were detected. In all six patients with a polyarticular presentation, laboratory tests for rheumatoid factor and ACPA were negative.

Conclusion: From the findings of this prospective, crystal-proven series of CPP crystal arthritis in an intrahospital setting, we can remark:

- 1. The low numbers of previous ambulatory flares may suggest a different clinical entity of CPP disease.

- 2. Radiological CC was absent in around a quarter of patients despite a extensive assessment, so synovial fluid analysis remains essential for diagnosis.

- 3. The rarity of associated metabolic diseases seen runs against ruling out secondary causes of CPP disease in this setting.

Disclosure of Interests: None declared

DOI: 10.1136/annrheumdis-2019-eular.7407

\section{SAT0443 RECONCILIATION OF URATE LOWERING THERAPIES DURING HOSPITALIZATION AND THE IMPACT OF RHEUMATOLOGIC CONSULTATION ON MANAGEMENT OF INPATIENT GOUT FLARES}

Mitali Sen. Emory University, Rheumatology, Atlanta, United States of America

Background: Hospitalizations complicated by gout flares have an impact on patient care. Delayed diagnosis and suboptimal management can lead to prolonged discomfort, impair chronic outcomes of the disease and lengthen the hospitalization (1). Patients on urate lowering therapy (ULT) are frequently admitted to the hospital for unrelated causes but there is variability in inpatient medication management of the urate lowering agent and acute management of the flare.

Objectives: In this descriptive study, we analyse the variability of reconciliation of ULT on admission and discharge and the impact of rheumatology consultation on acute and chronic management of gout.

Methods: Patients- above the age of 18- admitted to our tertiary care hospital from $01 / 01 / 2010$ to $01 / 01 / 2016$ with an ICD-9 or an ICD-10 diagnosis of gout were reviewed. The first 200 patients underwent a retrospective chart review as a pilot study for an ongoing project. We reviewed patient demographics, laboratory testing, and co-morbid conditions; medications on admission and discharge, incidence of gout flarediagnosis and management during hospitalization, rheumatology consultation and discharge plan for these patients.

Results: Of the 200 patients reviewed, 2.69 admissions per person. We further described the patients who had a gout flare during hospitalization ( $n=54,27 \%$ ). $66 \%$ of these patients were males, mean age 69.8 years and BMI $31.78 \mathrm{~kg} / \mathrm{m}^{2}$. A majority of patients had hypertension, renal disease, and dyslipidemia (Table 1)

$70 \%$ of the patients were on chronic medications for gout (Table 2) $29.6 \%$ of these patients were continued on these agents upon admission and only $64.8 \%$ of these patient was eventually discharged on these drugs.

Rheumatology consulted for $68.5 \%$ of the patients. Arthrocentesis was more frequently performed when rheumatology was consulted $(70 \%$ vs.17.6\%; $p<0.001)$. Rheumatology consultation did not decrease length of stay in the hospital. $78.5 \%$ of the patients managed by primary team were discharged on a ULT or colchicine compared to $100 \%$ in the group managed by rheumatology consult team $(100 \%$ vs $78.5 \%$; $<<0.0431)$. Outpatient rheumatology follow up was documented in discharge papers for $62 \%$ of the patients managed with rheumatology consult compared to $11.7 \%$ in the comparison group. (62\% vs $11.7 \%$; $<0.002$ ).

Conclusion: Rheumatology consultation improved adherence to guidelines in diagnosis and management of gout flare and improved the discharge planning and follow up. 